\title{
Ethical Frameworks in Public Health Decision-Making: Defending a Value-Based and Pluralist Approach
}

\author{
Kalle Grill $^{1} \cdot$ Angus Dawson ${ }^{2}$
}

Published online: 14 July 2015

(C) Springer Science+Business Media New York 2015

\begin{abstract}
A number of ethical frameworks have been proposed to support decision-making in public health and the evaluation of public health policy and practice. This is encouraging, since ethical considerations are of paramount importance in health policy. However, these frameworks have various deficiencies, in part because they incorporate substantial ethical positions. In this article, we discuss and criticise a framework developed by James Childress and Ruth Bernheim, which we consider to be the state of the art in the field. Their framework distinguishes aims, such as the promotion of public health, from constraints on the pursuit of those aims, such as the requirement to avoid limitations to liberty, or the requirement to be impartial. We show how this structure creates both theoretical and practical problems. We then go on to present and defend a more practical framework, one that is neutral in avoiding precommitment to particular values and how they ought to be weighted. We believe ethics is at the very heart of such weightings and our framework is developed to reflect this belief. It is therefore both pluralist and value-based. We compare our new framework to Childress and Bernheim's and outline its advantages. It is justified by its impetus to consider a wide range of alternatives and its tendency to direct decisions towards the best alternatives, as well as by the information provided by the ranking of alternatives and transparent explication of the judgements that motivate this ranking. The new framework presented should be useful to decisionmakers in public health, as well as being a means to stimulate further reflection on the role of ethics in public health.
\end{abstract}

Kalle Grill

kalle.grill@umu.se

1 Department of Historical, Philosophical and Religious Studies, Umeå University, 90187 Umeå, Sweden

2 Centre for Values, Ethics and the Law in Medicine (VELiM), University of Sydney, School of Public Health, Sydney, NSW 2006, Australia 
Keywords Decision-making - Ethical frameworks - Justification - Public health Value pluralism

\section{Introduction}

Ethical considerations often feature as a late addition to deliberations about policy options. When they do emerge, they are too often conceptualised as constraints on the pursuit of predetermined goals. We hold that ethical issues ought to be, on the contrary, at the heart of choosing policy goals, as well as deciding how those goals will be pursued. Choosing one alternative action or policy over another (including doing nothing) is always an ethical decision; at least in so far as such alternatives affect differently the wellbeing of other people.

In this paper we restrict our attention to the area of public health policy. What exactly constitutes 'public health' is open to dispute, but we suggest it is best to think about it as having a particular end in mind (improving population health) and certain kinds of paradigmatic behaviour (involving mass, collective activity). The focus on 'improving' population health should not be seen as being focused only on mere increase in the aggregate health of the population, as action to bring about greater equity in the distribution of health will also be relevant to this goal [19].

We believe that ethical frameworks can, in principle, be useful to policy makers and other decision-makers in public health contexts. However, they can also be problematic if they oversimplify or if they 'frame' decision-making with an inappropriate set of values [6]. Too often, the frameworks proposed for public health decision-making fail such tests: they can be incoherent and lacking in sufficient justification, it is unclear how they are to be used, or they yield counterintuitive recommendations [7]. In this paper we propose a new framework for thinking about public health policy, contrast it with a leading contender framework $[2,3]$, and justify the use of our framework by pointing to its advantages both in terms of the decisions likely to be made and the useful information produced and disseminated.

\section{The Framework}

There are many proposed ethical frameworks in the literature that might be applicable to public health decision-making [2, 3, 17, 18]. Each framework has slightly differing aims and content, and seeks to identify and promote particular ethical features. One common element to all these frameworks is that they propose substantive and particular ethical content. As an alternative to such frameworks, we propose what we call a value-based, pluralist framework. Our aim is not to contribute to either the philosophical debate on value pluralism or that on the nature of value, but rather to describe how a straight-forward form of pluralism (committed only to the modest view that there is more than one morally important value) can be coupled with common-sense decision-making based on such values. This can 
provide a framework that is both practically useful and has various advantages over other frameworks on offer.

Our framework has a number of distinguishing features in relation to the basis on which decisions are made. First, the framework allows that any important ethical consideration can help determine what decision is made. We use the word "value" broadly, to include the honouring of duties, the non-infringement of rights, and the development and expression of virtues. Second, in our framework, all values are 'equal', in the sense that none are assigned any special status prior to their use within the framework. This is taking to heart Charles Lindblom's insight that public decision-makers only confront values in increments and have little use for general value hierarchies [13, p. 83]. Third, public health measures typically affect the population as a whole, or large parts of it, and because they can impact on our lives in many different ways, public health decisions must consider a very wide range of values. Our framework is designed to facilitate the capture of all values relevant to a decision.

While the first feature makes for great inclusivity and accommodation of different moral perspectives, the second feature means that some moral perspectives are nonetheless excluded. These include any perspective on which there are absolute side-constraints. One might say that we are ecumenical concerning the sort of values that can affect decision-making, but rigid concerning the role those values play relative to other values. Accounts that focus on side-constraints run into fundamental problems, as we will see below.

For transparency and for illustration, some values that we would ourselves include in many public health decisions are individual health, population health, health equality, individual liberty, solidarity, social trust, and material wellbeing [for example as measured, if imperfectly, by Gross National Product (GNP)]. All these values can obviously strongly affect and be affected by public health measures, and they will clearly clash in some cases. Some method of weighing the different values against each other needs to be used. For example, individual liberty and material wellbeing may be affected in a situation where liberty-limiting measures such as detention, quarantine, and restrictions on travel and transportation are introduced for the sake of protecting the health of the population. As shown by these examples, values can be relevant for public health either because they can be promoted or because they can be diminished, and typically both can occur (individual liberty and material wellbeing can, for example, be both promoted or impeded by removing direct health threats).

Our framework consists of a series of explicit and formal steps to be taken as a means to reaching the best possible decisions about public health policy (or public health action). The steps are as follows:

1. Identification: Identify relevant alternatives.

2. Distinguishing: Distinguish relevant empirical differences between alternatives, including contingencies.

3. Ranking: Rank, as far as possible, alternatives from best to worst.

4. Evaluation: Make explicit, as far as possible, in what sense some alternatives are better than others. 
5. Documentation: Submit the result of the evaluation to a designated oversight institution.

For most decision-makers, it is natural to start with the available alternatives [13, p. 82]. We want to emphasise, however, that step 1 is a creative process. Although, typically, many alternatives will be obvious, this step requires much more than merely listing the measures most commonly used in similar situations. Insufficient attention to this step risks leaving good or optimal alternatives without consideration. Which alternatives are relevant depends in part on what particular problem or issue calls for a decision to be made. Some decisions are more long-term and strategic, in which case a wider array of alternatives will be relevant. Other decisions are more short-term and tactical, or focused, in which case the relevant alternatives will tend to be limited by the concrete issue at hand, though good alternatives can still seem surprising at first. To ensure that important alternatives are included, decision-makers should be inclusive and attentive to input from any stakeholders at this step in the process.

Step 2 involves an analysis of the inherent characteristics and consequences of each identified alternative. As in step 1, creativity is needed. More than in step 1, empirical expertise is essential. This step is probably the most familiar to decisionmakers. It concerns answering the common question "what would happen if we...?". As a rule, we cannot know for sure what consequences alternatives will have, but can only assess the likelihood of various possible outcomes. Judgments should be made on the basis of the quality of the evidence that is considered relevant and how it may be applied to the decision in hand.

Both step 1 and step 2 must be guided by judgments employing our values. We can aim to consider all possible alternatives and all the differences between them, but most of the time we will have to limit our attention to appealing alternatives and important differences. However, these necessary value judgments are provisional. They can be imprecise and open to error. They do not involve ranking, but only sorting into broad categories-appealing or not, important or not. At this stage, any disagreement or hesitation in relation to this sorting should be solved by being as inclusive as possible. In fact, it will often be wise to include all alternatives and differences that anyone either among decision-makers or other stakeholders consider appealing or important. This will make the explication or explanation in step 4 more comprehensive in the wider context in which the decision is made.

Though step 2 anticipates steps 3 and 4, it is useful to first distinguish the empirical differences between alternatives, because disagreements on these are of another type than disagreements on values (empirical disagreements can in principle be settled by science), though the two are easily confused. Empirical differences include differences in the likelihood that certain outcomes will ensue and differences in the magnitude of impacts that may otherwise be shared by two or more alternatives.

While step 2 can be very demanding, step 3 is perhaps even more so, since it involves both judgments on the relative importance of different values and judgments on the importance of risks and uncertainties (as identified in step 2). The demands are somewhat limited by focusing on the ranking of alternatives, without 
measuring their exact relative appeal. Sometimes, alternatives may defy any attempts at ranking. Two alternatives may seem equally good or on a par [1], or they may seem incomparable because they each favour a different value, where these values seem incommensurable [15, chapter 5]. In either of these circumstances, the ranking will be incomplete. If the ranking does not identify one alternative as the optimal alternative, it may make sense to take two or more alternatives on to step 4, to see if making the case for each alternative more explicit might help arbitrate between them. In the end, some alternative will have to be chosen, as we are dealing with concrete decisions that cannot be avoided.

Step 4 involves making it explicit in what sense the different possible alternatives are better or worse than others. These explanations must be phrased in terms of values. Minimally, they should identify what values are furthered or protected by what alternatives. Preferably, they should also identify what value comparisons motivate the ranking of alternatives in step 3. Such identifications or explanations will typically have the form "alternative A is better than alternative B because A has the cost $\mathrm{x}$ while $\mathrm{B}$ has the cost $\mathrm{y}$ and $\mathrm{x}$ is less important than $\mathrm{y}$ ", or, for a more complex case, "alternative A is better than alternative B because A likely yields the benefit $\mathrm{x}$ while $\mathrm{B}$ definitely yields the benefits $\mathrm{y}$ and $\mathrm{z}$, and a likelihood of $\mathrm{x}$ is more important than a certainty of $y$ and $z$ ". If incomparabilities are involved, the explanation may have the form "alternative A is incomparable with alternative $\mathrm{B}$ because $\mathrm{A}$ has the benefit $\mathrm{x}$ and $\mathrm{B}$ has the benefit $\mathrm{y}$, and $\mathrm{x}$ and $\mathrm{y}$ are incomparable [or incommensurable]". If top alternatives are on a par or incomparable, and so the outcome of the decision a result of mere picking, there should still be an explanation of this choice, of the form "we pick alternative A [B] because of... [some explanation in terms of positive features of alternative A [B] and/or negative features of alternative B [A]."

Explanations may not, for example, have the form "alternative A is better than alternative B because alternative A has cost $\mathrm{x}$ and this is unacceptable". Appeal to such a principle is not permitted within our framework because this kind of principle would pre-empt the issue of how we should evaluate the totality of relevant values in thinking about policy options. Such a neutral consideration of values is at the heart of the framework. We will discuss and defend this feature in the next section.

By putting step 3 before step 4 we want to leave room for the possibility that alternatives are ranked intuitively or based on a form of judgment that cannot be made fully explicit. We believe that alternatives are in fact often ranked in some such manner. We also do not want to exclude the possibility that intuitive or nonexplicit judgment is the epistemically most appropriate way of making moral judgments. 4 nevertheless of course requires that such judgment be made explicit to the greatest extent possible.

Steps 3 and 4 jointly include both the ranking of alternatives and the explanation for this ranking. Together these two steps correspond to various other decisionmaking processes which start from the properties of alternatives to deduce a preference among these alternatives. An important class of such decision-making processes are multiattribute decision analysis, as pioneered by Keeney and Raiffa [10]. We do not mean to exclude the use of such helpful tools, and remain ecumenical concerning the process by which the ranking of alternatives is produced. 
If the decision-maker uses a bottom-up approach like Keeney and Raiffa's to rank alternatives, this same approach will also supply much of the explanation of this ranking. Importantly, however, our framework is in no way dependent on the possibility of a complete inventory of considerations needed for these processes, nor the attribution to these circumstances of a precise relative importance.

Step 5 simply amounts to documenting the end result produced in step 4 . How exactly this is best done will depend on the particular circumstances, including the available resources of the decision-making agent or agency. The documentation should be submitted to some oversight institution that oversees the proper functioning of the public health agent, holding it accountable for its decisions, and for making decisions in accordance with the framework. This oversight institution could be the public. However, even if this is normally the case, this may not always be possible; for example, where information is retained from the public so as to avoid undesirable reactions, such as panic in the face of a pandemic. ${ }^{1}$

At steps 2, 3 and 4, new information and new insights are likely to surface, which may lead to the identification of additional relevant alternatives. This is an important part of the deliberative process. Decision-makers should continuously consider whether new information makes it appropriate to go back to a previous step and continue from there. This means that the steps, despite having a clear order, are not strictly chronologically separated. The decision-maker can return to an earlier step because of new evidence or a new perspective. However, the order of the steps is still important because it structures the deliberative process. For example, though thinking about the ranking of alternatives may lead one to notice additional alternatives or empirical differences between alternatives, which warrants going back a step or two, disagreement on the exact value of some alternative or empirical difference should not interfere with the identification of further alternatives or differences.

\section{Values or Constraints?}

By understanding public health decisions in terms of values which are all equal, we part ways with frameworks that understand some values as goals to be promoted and others as constraints on the promotion of such goals. One very influential such framework was presented by a collective of established scholars in 2002 [4]. Their general approach is to consider "how much weight and significance to assign to the ends and effects of protecting and promoting public health relative to the other considerations that limit and constrain ways to pursue such outcomes" [4, p. 171].

\footnotetext{
1 Step 5 is the closest we come to a publicity condition. Our modest commitment to publicity is one of the things that distinguishes our framework from Daniels and Sabin's Accountability for Reasonableness framework. Our framework also does not contain any steps corresponding to the relevance or the revision and appeals condition of Daniels and Sabin's framework [5, e.g. pp. 118, 119]. We do not engage with the issue of how the decision-maker should form her moral outlook, but limit ourselves to the issue of how she should decide based on the moral outlook she has. That said, we do believe that using our framework will promote transparency and accountability, as well as moral development or learning, as we will explain below.
} 
The concrete output is a list of five justificatory conditions "intended to help determine whether promoting public health warrants overriding such values as individual liberty or justice in particular cases" [4, p. 172]. There is, obviously, a very clear distinction made between goals and constraints. The first author behind this framework, James Childress, has gone on to develop a similar but more elaborate and more specific framework, in collaboration with Ruth Bernheim [2]. Childress and Bernheim [3] recommit to this framework, with minor modifications, in a further recent publication. We consider this framework to be the state of the art in this area and so will focus our critique on it and use it for purposes of contrast to further explain our own proposed framework.

Childress and Bernheim's framework, or, as they say, the "elements of a framework", is supposed to "apply both to the formation of public health policies and to public health officials' decisions within these indeterminate policies" [2, p. 159]. However, they limit their scope to "conflicts that arise in the selection of means to protect and promote public health" [2, p. 158]. It is, in other words, only the means aspect of the formation of policy that the framework addresses. As we will try to show, this focus on means, in isolation from goals, severely limits the applicability of the framework. Childress and Bernheim propose that the relevant issues around means concern, first, effectiveness and efficiency, and, second, "any ethical and other constraints that may apply to possible means" [2, p. 159]. The presumption is, in other words, that while public health goals are to be promoted, other values operate as constraints on the protection and promotion of these goals, rather than as desirable ends in their own right.

Childress and Bernheim do not list which values they believe operate as constraints rather than as ends, but it is obvious from their discussion that "liberty" is one of them. They call their framework "presumptivist" and explain that their presumption in favour of liberty can be rebutted under certain conditions. A libertylimiting decision will meet these conditions if

1. it has a "reasonable prospect of success",

2. it is "necessary and essential",

3 . it is the "the least restrictive and least intrusive alternative",

4. its "probable benefits (in risk reduction), minus any probable negative effects, are sufficient to rebut the presumption", and

5. it is "imposed impartially" [2, pp. 160, 161].

While the presumption in favour of liberty is merely a presumption, the conditions are presumably strict conditions for when that presumption is to be rebutted. Condition 2 and 3 are closely related, as Childress and Bernheim note, but they are not identical: While condition 2 requires that any limitation of liberty be necessary and essential, condition 3 requires that any limitation of liberty be as small as possible.

We see two major problems with this framework. The first concerns its applicability to real decisions and stems from conditions 2, 3 and 5. The second problem concerns an indirect reliance on a balancing of values the framework is officially designed to avoid. This problem stems from conditions 1 and 4 . 
Regarding the first problem, note first that, even if we have a rather specific goal in mind, there are typically different ways to achieve this goal and these different means will typically vary in what exactly they achieve and with what likelihood. Consider the goal that Childress and Bernheim use to illustrate their framework- to stop "the SARS outbreak or an avian influenza pandemic". Childress and Bernheim only consider and compare two means-forcible quarantine and requests for voluntary quarantine. It seems obvious that there are various other possible means to this end, including pre-vaccination and targeted use of antiviral agents (as discussed and compared with quarantine by Longini et. al. [14]). Another relevant measure to consider would be social distancing by cancelling events and closing schools and workplaces, etc. (on the effectiveness of social distancing generally see e.g. Glass et al. [9] on the use of social distancing to stop real pandemics, see e.g. Ferguson et. al. [8]). However, even if we accept a dichotomous choice between forcible quarantine and voluntary quarantine, it seems very likely that these two means will vary in likely outcomes and likelihood of outcomes within different contexts. Perhaps, for example, voluntary quarantine is more uncertain due to difficulty in estimating people's willingness to comply. Perhaps, on the contrary, forcible quarantine is more uncertain due to the risk of organized non-compliance as a reaction to what is perceived as an overly intrusive measure.

Now return to Childress and Bernheim's framework. Consider condition 2. This condition (as well as condition 3 and 5) have both a weak and a strong interpretation. On the weak interpretation, intrusions should be avoided when they serve no purpose in terms of goal-fulfilment. On this interpretation, means are "necessary and essential" if they are required to either increase the effectiveness of the goal or the likelihood of such a goal coming about. As Childress and Bernheim phrase it at one point, "other things [being] equal ... persuasion ... or other incentives ... should have priority over forcible detention" [2, p. 160]. Interpreted in this way, a draconian measure may be justified if it is only slightly more effective, or slightly less uncertain, than a very innocuous measure that may still be quite effective. For example, imposing a strict general curfew may be warranted to stop a pandemic, even if information and voluntary vaccination would also stop the pandemic, only somewhat more slowly. Such implications seem at odds with Childress and Bernstein's focus on the special status and importance of liberty.

On the alternative, strong interpretation of condition 2, means are "necessary and essential" if they are completely unavoidable. On this interpretation, intrusions should be avoided at any cost. For example, voluntary quarantine should be preferred to forced quarantine even if it is likely to be much less effective. This interpretation gives liberty (and impartiality) priority over other values. This, however, is not the flexible framework Childress and Bernheim [2, p. 160] claim to present, but rather an "absolutism" which they reject, arguing explicitly that giving priority to any value over any other "encounters devastating counterexamples" and is "unable to address all real-world complexities".

The strong interpretation would be more plausible if the only alternatives that are eligible are those that reach a particular goal, such as stopping the tragically real and ongoing Ebola outbreak. This would exclude both non-intrusive but ineffective alternatives and passivity. At points, it sounds like Childress and Bernheim mean 
their framework to be applied only to means to some very specific such goal. However, as already noted, alternative public health strategies or measures will always vary in the degree to and the likelihood with which they reach some generic goal. Suppose, for example, that quarantining 100,000 people will restrict further spread of the disease to below 1000 infections with a .8 probability, and quarantining 500,000 people will achieve the same result with a .9 probability. Or, similarly, that the smaller quarantine will in all likelihood restrict further infections to below 1000 while the larger will with the same likelihood restrict further infections to below 500. Now, what means are necessary? The framework cannot tell us this without prior specification of the goal in terms of, for example, some minimal probability for some maximal number of further infections. Such specification is hard work and, furthermore, will risk excluding alternatives that are almost as effective in terms of specific goal achievement but much less intrusive, or in other ways much better than the best eligible alternative. Therefore, moderation of the strong condition by restricting eligible alternatives makes the framework quite unhelpful.

It seems that neither the weak nor the strong interpretation is plausible. Various other interpretations are of course possible. One family of interpretations take the conditions to not really be conditions at all, but rather reminders of relevant considerations, rather like a list of values. However, such an interpretation is not only in strong tension with the explicit formulation of the conditions as conditions, it is also in tension with Childress and Bernheim's [2, p. 160] rejection of what they call "the contextual approach" which recommends that a decision-maker "simply balances all of the relevant values in a particular context".

At the risk of being pedantic, we note that there is in fact a further requirement built into condition 2. Decision-makers "must be able to provide strong reasons for their belief that a coercive approach is necessary". This is an epistemic condition, suggesting that there are stricter demands on certainty regarding the necessity of liberty-limiting means than that required regarding other things, such as for example the other conditions on the list, or regarding the possibility that liberty will be limited (perhaps rather weak reasons to believe that liberty will be limited are enough to rule out the promotion of individual health?). Childress and Bernheim say no more on the topic, so perhaps they do not intended a separate epistemic requirement but rather just mean to emphasise the importance of the central requirement in condition 2 .

Lacking a plausible interpretation of condition 2 for comparisons between means that differ in more than one dimension, we should perhaps conclude that the framework is not applicable to such comparisons. In some cases, there is no need to distinguish the strong and the weak interpretation. Perhaps the framework is only intended to be applied to such cases. These are cases where alternatives differ only in the extent to which they diminish liberty. Childress and Bernstein seem to have such a case in mind when they compare forced with voluntary quarantine as an illustration of condition 2: "It might be possible, for instance, to secure voluntary compliance with quarantine requests without resort to the threat or use of force" [ 2 , p. 160]. However, very few, if any, real alternatives will vary only in their degree of intrusiveness. For example, in their own chosen quarantine case, the two choices 
will almost certainly have differential impact upon the disease, so at a minimum we face a balancing of intrusiveness and effectiveness, not to mention possible differential impacts of degrees of intrusiveness upon the distribution of the disease in the population etc. etc. It would seem, in sum, that Childress and Bernheim's framework is either absolutist, or minimalist, or has no useful practical application.

We have focused our discussion on condition 2 but the requirement of condition 3 that "coercive measures should be necessary in degree as well as in kind" obviously has the very same structure and concerns the same value-liberty. The requirement of impartiality in condition 5 is also very similar. As with limitations of liberty, some values can be realized to a larger extent or with a greater likelihood if some partiality is allowed. For example, public health measures can target people based on characteristics listed by Childress and Bernheim as inappropriate: "race, ethnic background, socioeconomic class, or geographical location". Targeted measures may increase goal achievement because, statistically speaking, people with these characteristics may be more likely to benefit or more likely to pose a risk to others. Therefore, if this is the sort of partiality that condition 5 forbids, absolutism can be avoided only at the price of limiting the application of this condition to comparisons between alternatives that differ only in their degree of partiality.

It may be, however, that Childress and Bernheim use "partiality" in another sense. In discussing this condition, they denounce singling out people for blame based on the quoted broad characteristics. Since blaming is seldom an efficient method for reaching worthwhile goals (though it might be), absolutism may come rather cheap. Indeed, Childress and Bernheim note that condition 5 may seem "unnecessary or even useless", but they nevertheless defend it by pointing to persisting discrimination. We might therefore interpret this condition as requiring not impartiality in a wide sense, but only avoidance of unnecessary and unjust discrimination. This is reasonable enough. However, it is not practical to construct a framework by adding a presumption against all mistakes and moral failings that decision-makers are prone to, and if one would nonetheless try, the list would have much more than five items. Therefore, this weak, anti-discrimination interpretation of condition 5 arguably renders this condition redundant.

Before turning to the second major problem with Childress and Bernheim's framework, we should note, for completeness, that the framework is not necessarily applicable even to decisions between alternatives that differ only in the degree to which they entail limitations of liberty and partial treatment. This is because no guidance is given regarding the relative importance of these two values, and the values may be interdependent. Sometimes we can reach a goal either by using some force, or by being somewhat partial.

The second major problem with Childress and Bernstein's framework is that it reduces to a form of balancing of values, only one that is more complicated and obscure than the straightforward balancing that we favour. Childress and Bernstein's condition 1 requires "a reasonable prospect of success" [2, p. 160]. This could possibly be understood as imposing a set threshold probability of success for all public health measures, but this seems clearly unreasonable. In desperate situations, we may have to try measures with a probability of success lower than 
what we require of more everyday measures. Moreover, a lower prospect of success must be accepted for a very important goal which entails very minor intrusions than for a less important goal which entails greater intrusions. Besides, Childress and Bernheim do not propose a rigid threshold. Therefore, the only plausible interpretation is that what counts as a "reasonable prospect" depends on what is at stake-i.e. what can be achieved and at what cost, particularly in terms of liberty limitations. However, this is just to say that what is a reasonable prospect of success depends on what values are affected, in what way, with what probability. So while Childress and Bernstein's framework explicitly forbids balancing values against each other outright, it also demands that we balance them against each other in order to establish reasonable prospects of success.

Condition 4 is even more explicitly a balancing condition, requiring that "the probable benefits (in risk reduction), minus any probable negative effects, are sufficient" [2, p. 160]. This is most plausibly taken to mean that all possible benefits and costs should be considered, together with their associated probability, to ensure that the net expected effect is positive, perhaps by some margin. On this interpretation, condition 4 includes elements of all the first four steps of our preferred framework and amounts to a quite straight-forward balancing of values. The only alternative interpretation we can see emphasises the use of "probable" and takes this to mean that we should only consider benefits and costs which pass some threshold of probability. This, however, seems clearly unreasonable when considering improbable but catastrophic outcomes. Why should we disregard horrific possible consequences just because the likelihood of their realization is below some threshold? Moreover, it seems quite arbitrary to define this threshold, and quite unhelpful to have it once it is defined. One pictures decision-makers fiercely debating whether an outcome with an estimated $7 \%$ probability is probable or not probable, since this will make or break a public health proposal.

We have devoted substantial, perhaps excessive, attention to Childress and Bernheim's framework for public health decision-making. Much of our critique is admittedly directed at particular details of their framework. However, we boldly claim that any framework that operationalizes important values in the form of constraints on permissible means will face similar problems. At the very least, the problems we have identified with Childress and Bernheims's framework are hurdles to be overcome by any similar framework.

Intuitions to the effect that some means are impermissible typically point to a warranted concern that some value should be protected. However, it is hard to believe that any means have such enormous costs in terms of some value that they cannot be warranted by any gain in any other value. At least this goes for the values that are most obviously at stake in public health decisions. It may be that some means are always impermissible, whatever is at stake. Arguments to this effect have been presented in the case of torture, notably in a recent book by Kramer [11]. We assume that no such extreme means are at stake in public health decision-making, although some means will be more contentious than others.

We believe that ranking alternatives is part of everyday life as well as political and medical decision-making. The only way to avoid such ranking is to subsume every decision under some principle which does not in itself require ranking. We do 
not believe there are such principles for public health decision-making, or at least have not yet encountered one. Childress and Bernheim present two arguments against what they call the balancing or the contextualist approach: (1) it is too intuitive. (2) In real conflicts between the community and the individual, the community usually wins [2, p. 160]. Concerning 1 , we believe that by making explicit on what basis some alternatives are ranked higher than others, a decisionmaker learns about the underlying values and exposes her judgment to debate and scrutiny. This process will encourage decision-making that is based not on impulses but on considered judgments (call them intuitions or not).

Concerning 2, to the extent that it is true that the community usually wins in conflicts with individuals, this may well be appropriate given that the community consists of many individuals. When it is not appropriate, explicit attention to all values affected, including individual liberty, is the best way to get things right. It makes sense to us for a decision-maker to say that the forcible quarantine of even one single individual is not justified by minor reductions in health risks for very many others. It does not make any sense to us, however, to say that the forcible quarantine of one individual is justified if and only if it is the necessary, least restrictive and impartial means to the goal that we happen to have.

\section{Which Values?}

Childress and Bernheim presuppose that the only value to be promoted by public health actions is the unitary goal of public health. We have argued that the values which appear as constraints on the realization of that goal in Childress and Bernheim's framework are in fact values on a par with the value of public health, or perhaps with the set of values which are typically included under the heading "public health". Individual liberty is a value just like individual health is a value and they should both be given their due. Values can be either promoted or diminished by public health actions. Some values are more typically promoted and some more typically diminished, but no constant relationships should be assumed. A decision by an organization which has been given or has taken on the task of promoting and protecting public health may very well in some instances promote liberty and diminish health (for example by easing overly strict safety requirements). It may even be an explicit aim to promote liberty.

With this pluralistic view on the possible values that guide public health decision, are there any limits to what values may be relevant? It is consistent with our framework to restrict what values are eligible for consideration, as long as all eligible values are balanced against each other on equal terms. A restriction on eligible values may be motivated by practical concerns with coordination between various agents, including government agencies, and it may be motivated by normative concerns with what values it is appropriate to consider for agents which are focused on public health issues [16]. Though we recognize the value of coordination between people as well as between government agencies, we do not believe there are any general restrictions on what values are eligible for consideration in public health decision-making. 
It is possible that there may be constraints on what values a government should consider more generally, in any policy area. Perhaps a government should not be concerned with values that are too controversial [12]. We also recognize that there may be special weightings of values that apply generally. Perhaps a government should be more concerned with the health and wellbeing of their citizens or their residents, that with that of non-citizens or non-residents. We do not take a stand on these very general issues, but merely acknowledge them. We do presuppose, of course, in opposition to some political philosophies, that the government should be concerned with the health of at least some large segment of the population residing within its borders.

We further recognize that public health policy and public health work is regulated by law. We mean our framework to be used in the first instance by public health decision-makers acting within the limits of the law. At the same time, we believe that our framework makes it more acceptable to grant public health agencies relatively wide discretion to act within the boundaries set by a legitimate and democratic government. Typically, there are many things that public health agents are not explicitly required to do, but that they are not legally prevented from doing either. Since public health decisions should consider such values as individual liberty and material wellbeing, which are not necessarily related to health, there could theoretically be situations in which an agent or agency which is generally supposed to be promoting health instead seeks to promote some other value.

In a well-functioning society, there will be a complex system of different public agents charged with promoting and protecting various values. In such a context, it will rarely be best for a public health agency to use their time and resources to promote some value unrelated to public health, since this will interfere with the work of other agencies and will be less efficient than allowing each agency to do what it does best. However, in less fortunate circumstances, it may well be best for such an agency to promote values unrelated to public health. Consider a public health agency in a very poor country with a generally corrupt and incompetent government, where this agency is uncorrupted and efficient, perhaps thanks to direct support by some foreign benefactor. Should this agency stick to traditional public health work? Perhaps not. Perhaps it should build roads or irrigation systems. Perhaps it should do so even if sticking with traditional public health work would more obviously and directly benefit typical public health goals such as individual health. It may even be that it should do so at the price of diminished public health, though because of the broad scope of public health it is difficult to find examples. Consider a country in chaos, which is under threat from a foreign power that will enforce an authoritarian but very health-conscious regime. In this situation, a well organized public health agency should perhaps use its resources, such as its information dissemination networks, to prevent occupation, rather than use them to, for example, prevent disease. We do not see any absurdity or threat to the wellorderedness of society in admitting that there are or could be these unusual cases.

In sum, we do not see any principled limits to what values a public health agency should consider or promote, though in most circumstances practical considerations will entail that it should focus overwhelmingly on typical public health work. 


\section{Justification of the Framework}

We have explained how Childress and Bernheim's framework is unhelpful and impractical in several respects, while noting that our framework does not have these disadvantages. Instead of entangling decision-making in complicated multiple weighings or half-weighings as well as in the consideration of complicated conditions that require certain disvalues to be minimized or entirely avoided, we propose a straight-forward consideration of all relevant alternatives to be judged in terms of how they fare in respect to all relevant values. One major justification for our framework is that this approach simply directs decisions efficiently towards the best alternatives. However, the framework is further justified by the informational content conveyed by making decisions in this way.

Because step 4 in our framework involves explication of the reasons for a decision, in terms of values, and because step 5 involves making this explication or explanation available, any decision made under our framework will yield a dissemination of useful information. There will be an explanation, in terms of values, as to why one alternative is the preferred one. This explanation will contain information on what values and disvalues the decision-makers considered relevantly affected by the decision, and, to some extent, the relative importance of these effects. This information is useful because it can be generalized to the extent that decision-makers keep their moral outlook. Any other decision where values are affected in similar ways, though the empirical particulars may be different, can be expected to go the same way. For decisions where the highest ranked alternative is vastly superior, future decisions in similar cases will predictably go the same way even if there are some not insignificant changes in how other values are affected. If future decisions are like past ones except that some new alternative is available with more value or less disvalue relative to an earlier preferred alternative, it can be predicted that this new alternative will be chosen. And so on. Over time, the pattern of decisions made will clarify how the decision-maker compares different amounts of different values, and predictions can become more accurate. Assuming that the evaluations are transitive in regard to betterness or preferedness, a series of decisions that only partly overlap in what values are relevant will together yield substantial predictively useful information. Daniels, discussing his similar publicity condition, notes how "the pattern of such decisions will resemble a type of "case law'" [5, p. 121].

To illustrate, if a public health official who follows our framework decides to detain 100 people for 2 weeks in order to save one statistical life, we may assume that she would do the same to save more than one life (other things being equal). If she then decides to accept a low risk of non life-threatening but cumbersome disease to $10,000,000$ people rather than detain 100 people for 2 weeks, we may assume that she would accept the same risks in order to save one statistical life. In reality, alternatives will seldom be identical in this way, but they will sometimes be relevantly similar and with some contextual interpretation, decisions and explanations will convey a rich body of information in the aggregate. 
Contrast this with a public health official who uses a framework which tells her to prefer the least restrictive alternative. Assume that this official decides to detain 100 people for 2 weeks because this is the least restrictive way of saving one statistical life. This conveys very little information on what to expect from this decision-maker in other, future decisions. We may of course predict that she will choose the least restrictive alternative, but this may sometimes be very restrictive. Perhaps next time the least restrictive way of saving one statistical life is to detain 10,000 people for 2 months. Regardless of how many decisions are made based on this rule, we will never know how much restriction we may expect in order to achieve any particular goal. Childress and Bernheim's framework is, of course, richer than the one condition of preferring the least restrictive alternative. Deliberation on conditions 1 and 4 in their framework will convey useful information. However, this is only because and only to the extent that these conditions incorporate elements of the balancing of values.

In general, that a decision meets a number of conditions conveys little information beyond this very fact. A large set of decisions based on Childress and Bernheim's conditions 2, 3 and 5 will teach us what the decision-maker means by intrusion and by partiality, but not how those disvalues compare with other values and disvalues. In particular, when you disagree with a decision made according to a list of conditions structured within a framework, there is not much to learn from further discussion and explanation. Supposedly, explaining a decision made according to such a framework means explaining how the preferred alternative meets the conditions. It does not mean explaining why those conditions are important and it does not mean motivating why any particular alternative (among those which meet the conditions-which may be many) is to be preferred.

Motivating a value-based decision, in contrast, means explaining the importance of the affected values and disvalues, and how they relate to each other. The public health official in the above example may explain that 2 weeks of detention for 100 people adds up to 200 weeks or approximately 4 years and that this is less than the loss of quality time from one early death. Or, less technically, she may explain how early death is an ultimate tragedy and how it impacts on family, friends and society, and compare that with the relatively mild effects of detention imposed for public health reasons. If she has used comprehensive multiattribute utility analysis, she may provide rich tables of considerations with their relative importance. Whatever the decision-maker says to explain her value comparison, it will tell us much about the relative importance she attributes to different values. This sort of information will help our predictions concerning how she will decide in completely different situations, where some of the same values are affected.

Since, as noted above, the predictive value of the information conveyed on a value-based approach is dependent on the consistency of the moral outlook of the decision-maker, decision-makers should arguably declare when they change this outlook, either as this happens, or in connection to the explication of new decisions that are not in line with earlier decisions. This requirement may be considered an integrated part of step 4, but was not listed above since it may alternatively be handled outside of the decision-making process. List-of-conditions-type frameworks, such as Childress and Bernstein's are similarly dependent on the conditions, 
and their interpretations, remaining unchanged. However, it may perhaps be an advantage of this approach that changes in moral outlook will lead to changes in the list of conditions, which are potentially more obvious than changes in values.

We have focused on how information about the basis on which decisions are made can help us to predict future decisions. Prediction, however, is only the most obvious use of such information. Its availability is also a prerequisite for transparency of decision-making. If the only information available regarding a decision is that it meets a number of conditions, transparency is rather superficial. If, on the other hand, alternatives are ranked and the ranking motivated in terms of all relevant values, many detailed considerations will be open to scrutiny and evaluation. This rich information also makes possible much more comprehensive responsibility ascriptions. Under a value-based framework, decision-makers can be held responsible for the detailed value judgments they make, and not only for their final decisions. Under a list-of-conditions-type framework, a decision-maker can only be held responsible for her interpretation and application of the list of conditions. Transparency and responsibility are of course in turn vital to democracy and healthy meritocracy.

Finally, value-based decision-making naturally facilitates learning from previous judgments. A simple way to show this is to point to the greater occasion for errorthe best alternative may have been preferred, but there may still be mistakes in the ranking of non-optimal alternatives. Under a list-of-conditions-type framework, in contrast, only the decision itself can be either correct or incorrect. Furthermore, an incorrect decision under a list-of-conditions-type framework is just that, mistakes are either/or, they do not allow for degrees of correctness. Value-based decisions, in contrast, can be mistaken to the degree that the relative importance of any one of two values is exaggerated or downplayed.

\section{Conclusion}

We have argued that our framework is justified by the outcomes of using it. This is intended as a form of mid-level justification. We do not take a stand on issues of ultimate justification, and it would be unnecessary to do so in this context. Childress and Bernheim propose that the use of the police power "requires moral justification that the public in whose name the policies are carried out could reasonably be expected to accept" [2, p. 158]. This contractarian approach is one way to provide a fundamental justification. We are happy to accept it provisionally but do not exclude alternatives. However, in disagreement with Childress and Bernheim, we believe that all decisions require justification, not just those that might reduce liberty.

By facilitating prediction and promoting transparency and comprehensive responsibility, as well as learning and development, our value-based framework yields positive effects through its very application, and these positive effects are such that they will tend to make for increasingly higher quality decisions. This strengthens the propensity of the framework to direct decisions towards the best alternatives. This tendency depends more fundamentally on the framework's 
inclusive focus on all relevant values, understood as formally equal, without vague presumptions and other unnecessary complications.

Acknowledgments We are grateful to participants at the seminar in Medicine, Ethics, Society and History at the University of Birmingham for helpful discussion of an early draft, and to participants at the seminar at the Centre for Advanced Studies in Bioethics in Münster for a very fruitful discussion of a later draft. Grill's research was funded by the Swedish Research Council for Health, Working Life and Welfare, under project 2009-2189.

\section{References}

1. Chang, R. (1997). Incommensurability, incomparability, and practical reason. Cambridge: Harvard University Press.

2. Childress, J. F., \& Bernheim, R. G. (2008). Public health ethics-Public justification and public trust. Bundesgesundheitsblatt-Gesundheitsforschung-Gesundheitsschutz, 51(2), 158-163.

3. Childress, J. F., \& Bernheim, R. G. (2013). Introduction: A framework for public health ethics. In R. G. Bernheim, J. F. Childress, R. J. Bonnie, \& A. L. Melnick (Eds.), Essentials of public health ethics. Burlington, MA: Jones and Bartlett.

4. Childress, J. F., Faden, R. R., Gaare, R. D., Gostin, L. O., Kahn, J., Bonnie, R. J., et al. (2002). Public health ethics: Mapping the terrain. The Journal of Law, Medicine \& Ethics, 30(2), 170-178.

5. Daniels, N. (2007). Just health: Meeting health needs fairly. Cambridge: Cambridge University Press.

6. Dawson, A. (2009). Theory and practice in public health ethics: A complex relationship. In S. Peckham \& A. Hann (Eds.), Public health ethics and practice (pp. 191-210). Bristol: The Policy Press.

7. Dawson, A. (2011). Resetting the parameters: Public health as the foundation for public health ethics. In A. Dawson (Ed.), Public health ethics: Key concepts and issues in policy and practice (pp. 1-19). Cambridge: Cambridge University Press.

8. Ferguson, N. M., Cummings, D. A. T., Cauchemez, S., Fraser, C., Riley, S., Meeyai, A., et al. (2005). Strategies for containing an emerging influenza pandemic in Southeast Asia. Nature, 437, 209-214.

9. Glass, R. J., Glass, L. M., Beyeler, W. E., \& Min, H. J. (2006). Targeted social distancing design for pandemic influenza. Emerging Infectious Diseases, 12, 1671-1681.

10. Keeney, R. L., \& Raiffa, H. (1976/1993). Decisions with multiple objectives: Preferences and value trade-offs. Cambridge: Cambridge University Press.

11. Kramer, M. H. (2014). Torture and moral integrity: A philosophical enquiry. Oxford: Oxford University Press.

12. Larmore, C. E. (1987). Patterns of moral complexity. Cambridge: Cambridge University Press.

13. Lindblom, C. E. (1959). The science of 'muddling through'. Public Administration Review, 19(2), 79. doi:10.2307/973677.

14. Longini, I. M., Nizam, A., Xu, S., Ungchusak, K., Hanshaoworakul, W., Cummings, D. A. T., \& Halloran, M. E. (2005). Containing pandemic influenza at the source. Science, 309, 1083-1087.

15. Richardson, H. S. (1994). Practical reasoning about final ends. Cambridge: Cambridge University Press.

16. Rothstein, M. A. (2002). Rethinking the meaning of public health. The Journal of Law, Medicine \& Ethics, 30(2), 144-149.

17. Tannahill, A. (2008). Beyond evidence-To ethics: A decision-making framework for health promotion, public health and health improvement. Health Promotion International, 23, 380-390.

18. ten Have, M., de Beaufort, I. D., Mackenbach, J. P., \& van der Heide, A. (2010). An overview of ethical frameworks in public health: Can they be supportive in the evaluation of programs to prevent overweight? BMC Public Health, 10, 638.

19. Verweij, M., \& Dawson, A. (2007). The meaning of 'public' in 'public Health'. In A. Dawson \& M. Verweij (Eds.), Ethics, prevention, and public health (pp. 13-29). Oxford: Oxford University Press. 